to investigate the association between the attempt and sociodemographic, behavioural and clinical variables.

Methods Data collected in 2008 from PASSI, the Italian behavioural risk factor surveillance system based on telephone interviews of residents aged $18-69$ years, were analysed to identify factors associated to quit smoking attempts.

Results Sample analysed included 18281 current and ex-smokers. Around two out of three people (11881) tried to quit smoking and among them 3911 (34\%) failed the attempt.

A multivariate analysis showed that smoking cessation attempt was more likely among males, elder (50-69 years) and married people; among those living with children ( $<14 \mathrm{yrs}$ ), with a temporary job, without economic difficulties and with a high level of education; among those with cardiovascular risk factors (hypertension, hypercholesterolaemia, obesity) and those affected by chronic diseases (almost one among cancer, diabetes and myocardial infarction). On the other side, sedentary people and those with good perceived health status are less intentioned to quit.

Stratification by sex showed a different strength of association between the outcome variable and other variables in males and females, but most important that education and perceived health status are not associated with the attempt to quit smoking among women.

Conclusions Population-based surveillance system tracking the characteristics of ex and current smokers, who tried to quit, may offer a lot of information, otherwise not available, for planning, implementing and evaluating promotion and prevention interventions to support people who try to quit smoking.

\section{P35 OBJECTIVE AND SUBJECTIVE METHOD OF PHYSICAL ACTIVITY MEASUREMENT IN A CROSS-SECTION OF ENGLISH ADULTS: HEALTH SURVEY FOR ENGLAND 2008}

doi:10.1136/jech.2010.120477.35

M Chaudhury, E Stamatakis, M Roth, J Mindell. Department of Epidemiology and Public Health, UCL, London, UK

Objective To describe and compare physical activity levels, sedentary and moderate-to-vigorous physical activity (MVPA) behaviour in HSE2008 in a representative sample of the adult population, using both objective (accelerometry) and subjective (self-report via questionnaire) methods of measurement.

Design Nationally representative cross-sectional population data from Health Survey for England, 2008.

Setting Random sample of the general population living in private households in England

Participants Subsample of 4507 adults aged $16+$ were selected for accelerometry wear, of whom 2115 adults had valid accelerometry data wear (with at least $600 \mathrm{~min}$ per day).

Main Outcome Measure Prevalence of those adults who meet the current physical activity (PA) recommendations by accelerometry data. Mean sedentary (minutes) and MVPA minutes (accelerometry).

Results Based on accelerometry data, $6 \%$ of men and $4 \%$ of women met the Chief Medical Officer's current minimum recommendations for PA by achieving at least 30 min of moderate or vigorous activity on at least 5 days in the week of accelerometer wear. Men and women aged 16-34 were most likely to have met the recommendations ( $11 \%$ and $8 \%$, respectively). In contrast, based on self-report measures, $39 \%$ of men and $29 \%$ of women were said to have met the PA recommendations. Only $10 \%$ of men and $8 \%$ of women whose self-reported activity level corresponded with meeting the recommendations also met the recommendations based on accelerometry. Overall, men had significantly longer periods of sedentary time per day than women (595 min and $584 \mathrm{~min}$, respectively), ( $p=0.003)$. While men spent an average of $31 \mathrm{~min}$ in MVPA in total per day, and women an average of 24 min, most of this was sporadic activity. Those who were not overweight or obese spent few minutes on average in sedentary time $(591 \mathrm{~min}$ for men and $577 \mathrm{~min}$ for women) than those who were obese (612 $\mathrm{min}$ for men and 585 for women). Similarly, adults not overweight or obese spent more MVPA minutes than those who were overweight or obese. This pattern was similar with each BMI category.

Conclusion Subjective self-reported method of assessing physical activity resulted in higher levels of activity than objective accelerometry data. Despite this, the results from objective accelerometer data corroborate self-report findings across age and sex. Objective measures provide more accurate data and should be used where available. Comparison between objective and subjective methods indicates that people over estimate their actual physical activity levels.

\section{P36 DISTRIBUTION OF PHYSICAL ACTIVITY AMENITIES IN SCOTLAND BY SMALL AREA MEASURES OF DEPRIVATION AND URBANICITY}

doi:10.1136/jech.2010.120477.36

${ }^{1} \mathrm{~K}$ E Lamb, ${ }^{1} \mathrm{~A}$ Ellaway, ${ }^{2} \mathrm{~N}$ S Ferguson, ${ }^{2} \mathrm{Y}$ Wang, ${ }^{3} \mathrm{D}$ Ogilvie. ${ }^{1} \mathrm{M}$ RC Social and Public Health Sciences Unit, Glasgow, UK; ${ }^{2}$ Department of Civil Engineering, University of Strathclyde, Glasgow, UK; ${ }^{3}$ MRC Epidemiology Unit and Centre for Diet and Activity Research (CEDAR), Cambridge, UK

Objective Obesity and physical activity are associated with aspects of the physical environment where people live, but the precise mechanisms of these associations are not well understood. Knowledge of the extent to which access to opportunities for physical activity is socially patterned is important to inform policy. A few studies of cities and regions (mostly conducted outside the UK) have found fewer resources in deprived areas, while others have not. The aim of this study was to examine the distribution of physical activity facilities by area level deprivation in Scotland, adjusting for differences in urbanicity and population density and specifically examining differences between Scotland's four largest cities.

Design A SportScotland data set, containing information about all Scottish sports facilities ( $\mathrm{n}=10283)$, was linked to Scottish Neighbourhood Statistics data on population size, deprivation category and urban classification for Scottish data zones. Poisson and negative binomial multilevel models were used to examine the association between facilities and deprivation quintiles, adjusting for random variation between local authorities.

Setting Scotland.

Results Prior to adjustment for urbanicity, the density of all facilities fell with increasing deprivation from quintiles 2 to 5 . After adjustment for urbanicity and local authority, the effect of deprivation remained significant but the pattern altered, with data zones in quintile 3 having the highest estimated mean density of facilities (2.63/1000 residents). The lowest estimated densities were observed in the most affluent $(1.99 / 1000)$ and the most deprived $(1.87 / 1000)$ quintiles, with no significant difference between these quintiles. After adjusting for population size, Dundee and Edinburgh had the highest estimated density of facilities whilst Glasgow had the lowest.

Conclusions Facility presence is dependent upon area level deprivation, after taking into account differences between local authorities and urban classification, with both the least and most deprived areas showing the lowest rates of facilities. The distribution of facilities across Scotland does appear to be socially patterned, but not according to a straightforward social gradient. There is a need to go further and take transport accessibility into account. 